\title{
A yoga intervention for type 2 diabetes risk reduction: a pilot randomized controlled trial
}

\author{
Kelly A McDermott ${ }^{1 *}$, Mohan Raghavendra Rao ${ }^{2}$, Raghuram Nagarathna ${ }^{2}$, Elizabeth J Murphy ${ }^{3}$, Adam Burke $^{4}$, \\ Ramarao Hongasandra Nagendra ${ }^{2}$ and Frederick $\mathrm{M} \mathrm{Hecht}^{1}$
}

\begin{abstract}
Background: Type 2 diabetes is a major health problem in many countries including India. Yoga may be an effective type 2 diabetes prevention strategy in India, particularly given its cultural familiarity.

Methods: This was a parallel, randomized controlled pilot study to collect feasibility and preliminary efficacy data on yoga for diabetes risk factors among people at high risk of diabetes. Primary outcomes included: changes in BMI, waist circumference, fasting blood glucose, postprandial blood glucose, insulin, insulin resistance, blood pressure, and cholesterol. We also looked at measures of psychological well-being including changes in depression, anxiety, positive and negative affect and perceived stress. Forty-one participants with elevated fasting blood glucose in Bangalore, India were randomized to either yoga $(n=21)$ or a walking control $(n=20)$. Participants were asked to either attend yoga classes or complete monitored walking 3-6 days per week for eight weeks. Randomization and allocation was performed using computer-generated random numbers and group assignments delivered in sealed, opaque envelopes generated by off-site study staff. Data were analyzed based on intention to treat.
\end{abstract}

Results: This study was feasible in terms of recruitment, retention and adherence. In addition, yoga participants had significantly greater reductions in weight, waist circumference and BMI versus control (weight $-0.8 \pm 2.1$ vs. $1.4 \pm$ 3.6, $p=0.02$; waist circumference $-4.2 \pm 4.8$ vs. $0.7 \pm 4.2, p<0.01$; BMl $-0.2 \pm 0.8$ vs. $0.6 \pm 1.6, p=0.05$ ). There were no between group differences in fasting blood glucose, postprandial blood glucose, insulin resistance or any other factors related to diabetes risk or psychological well-being. There were significant reductions in systolic and diastolic blood pressure, total cholesterol, anxiety, depression, negative affect and perceived stress in both the yoga intervention and walking control over the course of the study.

Conclusion: Among Indians with elevated fasting blood glucose, we found that participation in an 8-week yoga intervention was feasible and resulted in greater weight loss and reduction in waist circumference when compared to a walking control. Yoga offers a promising lifestyle intervention for decreasing weight-related type 2 diabetes risk factors and potentially increasing psychological well-being.

Trial registration: ClinicalTrials.gov Identified NCT00090506.

Keywords: Yoga, Prediabetes, Type 2 diabetes, India, Randomized controlled pilot

\section{Background}

Type 2 diabetes mellitus (T2DM) is a major global health problem with a prevalence of 366 million in 2011 that is projected to increase by $51 \%$, reaching 552 million by $2030[1,2]$. India follows this global trend with a prevalence of 60 million in 2011 that is projected to increase by

\footnotetext{
*Correspondence: mcdermottk@ocim.ucsf.edu

'Osher Center for Integrative Medicine, University of California, 1545 Divisadero St., San Francisco, CA 94115, USA

Full list of author information is available at the end of the article
}

63\%, reaching 98 million by 2030 [1]. Factors contributing to the high prevalence of T2DM in India include genetic predisposition exacerbated by environmental factors including increasing prosperity and urbanization resulting in increased abdominal obesity and insulin resistance $[3,4]$.

In India, there is also a high prevalence of prediabetes, with elevated fasting blood glucose (FBG) in the range of 5.6-6.9 $\mathrm{mmol} / \mathrm{L}[3,5]$. Individuals with prediabetes are at an increased risk of developing T2DM (FBG $\geq 7 \mathrm{mmol} / \mathrm{L}$ ); $5-10 \%$ will develop T2DM within one year, $25 \%$ within 
5 years [6]. Metabolic syndrome is another characterization of high risk individuals with at least three of the following five risk factors: elevated FBG, elevated blood pressure, low high-density lipoproteins (HDL), elevated triglycerides and abdominal obesity [7]. Lower cutpoints for waist circumference plus additional criteria for body mass index (BMI) and truncal subcutaneous fat have been recommended as criteria for metabolic syndrome in the Indian population based on their predisposition for T2DM and cardiovascular disease (CVD) [4,8].

Lifestyle interventions including exercise have been effective in offsetting T2DM complications and the progression from prediabetes or metabolic syndrome to T2DM [6,9-11]. By modifying muscle fibers and enhancing beta cell functions, exercise can optimize insulin sensitivity and improve glucose intolerance [12,13]. Thus, exercise may be particularly effective in earlier disease stages and has been associated with a 30\% reduction in metabolic syndrome [14] and a T2DM risk reduction of $63-65 \%$ in people with prediabetes [15].

In India there is a rich history of using yoga to manage T2DM [3]. Yoga is a mind/body practice based on traditional Indian philosophy, often incorporating three major components: held or sequences of physical postures, breathing exercises and meditation [16,17]. Yoga's energy expenditure is similar to other low to moderate exercise [18-20] and a recent review found yoga had beneficial outcomes similar to those of moderate exercise in populations with T2DM [21]. Subsequent to the current pilot study, members of our group completed a trial of $n=277$ participants in Bangalore, India with T2DM, comparing a yoga program aimed at T2DM management with an exercise program of comparable intensity. In that study yoga was similar to exercise in terms of reducing FBG, hemoglobin $\mathrm{A} 1 \mathrm{c}(\mathrm{HbA} 1 \mathrm{c})$, triglycerides, total cholesterol and very lowdensity lipoproteins (VLDL) [22]. Evidence suggesting that yoga may also be an effective preventive practice in high risk populations, such as with prediabetes and/or metabolic syndrome, is also accumulating [23]. Investigators recently concluded a large randomized controlled trial of restorative yoga vs. stretching for participants with metabolic syndrome and found that the yoga group had significant reductions in FBG that were sustained at 12 months [24].

Additional benefits of yoga include improved exerciserelated self-efficacy [25] quality of life [26] and mood $[27,28]$ all of which are important factors in maintenance of lifestyle behavior change. Yoga also may have a different effect on the sympathetic nervous system (SNS) and hypothalamus-pituitary-adrenal (HPA) axis response to stress compared to other types of exercise. Where other exercise can stimulate the SNS/HPA axis, yoga may be down regulating it, shifting to parasympathetic dominance of the stress response [21,23]. In Nagarathna et al., authors found that yoga was superior to exercise at decreasing the need for oral hypoglycemia medications, decreasing lowdensity lipoproteins (LDL) and increasing HDL, suggesting a benefit associated with yoga in addition to that of just increasing exercise [22].

The current pilot study compares yoga to a walking control among Indians in Bangalore over an 8-week intervention period. This study is a product of an international collaboration to share areas of expertise between the Swami Vivekananda Yoga Anusandhana Samsthana (SVYASA) in Bangalore, India and the Osher Center for Integrative Medicine (OCIM) at the University of California, San Francisco (UCSF). The pilot study aimed to establish feasibility based on meeting recruitment, retention and adherence goals and to collect preliminary efficacy data on changes in T2DM risk factors and psychological well-being.

\section{Methods}

\section{Study design and participants}

This was a 1:1 randomized controlled pilot study conducted in Bangalore, India. The study was reviewed and approved by the research ethics committees at SVYASA and UCSF. Written, informed consent was obtained from all participants. Figure 1 depicts a flow diagram of study screening, enrollment and analysis. Participants were recruited over one week, (October 24, 2004 - November 1, 2004) using advertisements placed in primary care and diabetes clinics, as well as strategic locations throughout the city. Advertisements invited individuals with a firstdegree relative with T2DM and interest in participating in a yoga program to contact the study staff to schedule a screening visit. Individuals were asked to come to up to two screening visits. The first visit evaluated FBG using a glucometer and finger stick capillary whole blood. Those with elevated FBG ( $\geq 5.6 \mathrm{mmol} / \mathrm{L})$ at the first visit were invited back for a second screening visit. During the second screening visit the glucometer assessment of FBG was repeated to confirm eligibility and an oral glucose tolerance test (OGTT) was performed. The OGTT used a standard 75 gm glucose load dissolved in $300 \mathrm{ml}$ of water ingested over 5 minutes. Glucose was then measured in laboratory assessments of venous blood. OGTT between 7.8-11 mmol/L was in the eligible range. Sixteen percent of participants did not return for the OGTT two-hour blood draw. Because all of these participants had a second FBG measure, the second FBG was used for screening purposes rather than the OGTT.

In addition to having two screening $\mathrm{FBG} \geq 5.6 \mathrm{mmol} / \mathrm{L}$, inclusion criteria for this study also included: 1) a firstdegree relative with T2DM; 2) age between 30-65 years; and, 3) willingness to participate in an 8-week yoga program. Exclusion criteria included: 1 ) an inability to provide informed consent; 2) taking medications associated with insulin resistance (e.g. glucocorticoids, thiazide diuretics, 


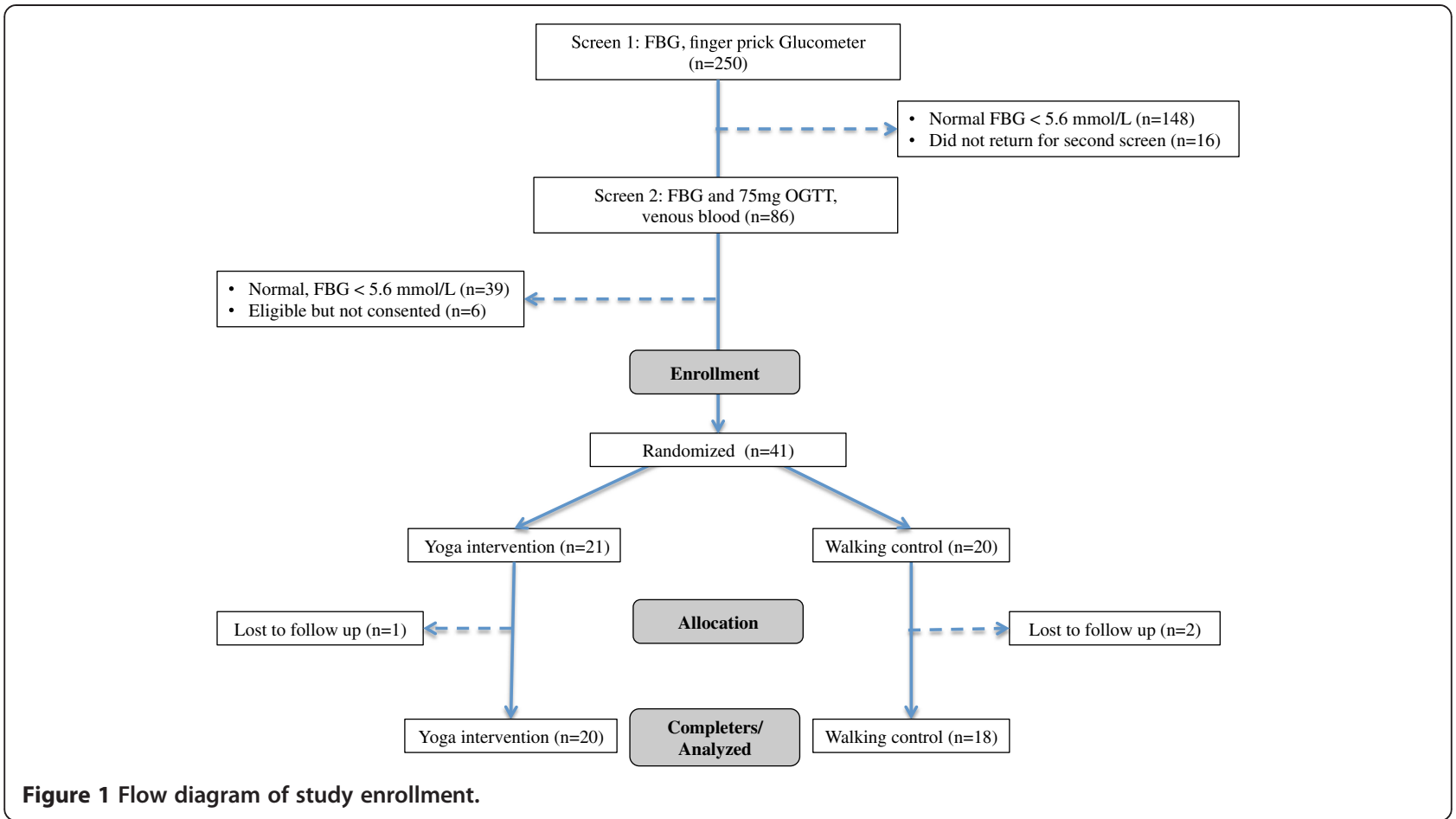

nicotinic acid, $\beta$-blockers); 3 ) prior diagnosis of diabetes or treatment with diabetes medications; 4) active liver disease (with AST or ALT > 3 times the upper limit of normal); 5) anemia with hemoglobin $<85 \mathrm{~g} / \mathrm{L} ; 6$ ) pregnant or plans to become pregnant during the course of the study; 7 ) recent major trauma or surgery that would interfere with participation; and 8) inability to speak either English or Kannada (the local language in Bangalore). Six participants with borderline FBG screening levels were mistakenly enrolled in the study and randomized. These participants are included in the main intention to treat analysis (ITT), but are excluded in the per-protocol analysis. The per-protocol analysis only includes participants meeting the inclusion criteria of having screening $F B G \geq 5.6 \mathrm{mmol} / \mathrm{L}$.

\section{Sample size and randomization procedures}

Based on this study's primary feasibility and preliminary efficacy outcomes, sample size was based on resources available. Randomization was performed using computergenerated random numbers and group assignments delivered in sealed, opaque envelopes generated by off-site study staff. Participants were not blinded to their group assignment.

\section{Intervention}

The yoga group attended a day long (eight hour) group counseling session on healthy lifestyle changes with topics on healthy diet, increasing physical activity and smoking cessation. Spouses were invited to attend this group counseling session as well. Participants in the intervention group were then asked to attend at least three, and up to six, yoga classes per week over the eight weeks of the study. Yoga classes were offered on six days of each week. The classes were held in a community hall and taught by two registered Ayurveda medical practitioners with Masters level yoga training from SYVASA. While one instructor demonstrated postures for the class, the other instructor adjusted individual participant's postures or gave them suitable alternative postures if necessary.

The yoga intervention was designed to manage glucose levels by increasing metabolism, reducing stress and facilitating a positive outlook. In addition, the classes sought to connect participants with a sense of responsibility and control over their health. Each class lasted 75 minutes total and had the following components: diabetes and stress management education (10 minutes); breathing exercises (6 $\mathrm{min})$; loosening exercises (10 $\mathrm{min})$; standing poses ( $8 \mathrm{~min})$; supine poses $(8 \mathrm{~min})$; prone poses $(8 \mathrm{~min})$; sitting poses ( $8 \mathrm{~min})$; relaxation/corpse pose $(6 \mathrm{~min})$; chanting exercises and seated meditation (10 $\mathrm{min}$ ) (see Table 1 for a more detailed account of postures). The total active time in poses was approximately 32 minutes. Evidence suggests that these poses specifically improve muscle metabolism and stress response [29] and the breathing exercises enhance basal metabolic rate [30,31].

The yoga intervention was manualized for this study and while yoga instructors received detailed instruction from study staff on how the class was to be conducted, the yoga classes were not observed by study staff. Participants were asked to do a home practice if they were unable to 
Table 1 Yoga class material and asana sequencing

1. Didactics-10 minutes

Diabetes causes, complications and lifestyle factors

Principles, philosophy, and practices related to a yoga based

lifestyle program

Stress response

Maladaptive behavior and behavior change

Emotion and coping

2. Pranayama (Breathing exercises) - 6 minutes

Hands stretch breathing

Ankle stretch breathing

Tiger breathing

Rabbit breathing

3. Loosening exercises (any 3) - 10 minutes

Jogging

Forward, backward, side bending

Twisting

Pavanamuktasana kriya (Supine knees to chest)

Surya namaskara (3 sun salutations)

4. Standing asana (any 3 ) -8 minutes

Padahasthasana (Foot hand)

Ardhachakrasana (Half moon)

Trikonasana (Triangle)

Parshvakonasana (Side angle)

Ardhakati chakrasana (Half wheel)

Vrikshasana (Tree)

6. Supine asana-8 minutes

Sarvangasana (Shoulder stand)

Halasana (Plough)

Matsyasana (Fish)

Pavanamuktasana (Supine knee chest position)

Naukasana (Boat)

7. Prone asana-8 minutes

Bhujangasana I, II , III (Cobra)

Shalabhasana - alternate legs, both (Locust)

Dhanurasana (Bow)

Navasana (Boat)

8. Sitting asana-8 minutes

Paschimottanasana (Seated forward bend)

Vakrasana/ardhamatsyendrasana (Half twist)

Ustrasana (Camel)

Sashankasana (Rabbit)

9. Relaxation Shavasana (Corpse) with guided scan-6 minutes

10 Chanting 'OM' monosyllables, primordial sounds in Indian philosophy attend at least three classes in a given week and were asked to keep track of each home practice in a daily diary.

\section{Control}

The control group attended the same day long (eight hour) group counseling session on lifestyle changes with their spouses. The control group was asked to do $30 \mathrm{mi}-$ nutes of walking plus breaks for rest on three to six days per week during each of the eight weeks of the study. Including rest breaks, the total time was approximately equivalent to the 75 minute yoga class. They were asked to perform their walking in the park adjacent to the community hall where the daily yoga classes were held. Two study volunteers monitored control group participants' attendance at daily walks in the park.

\section{Measures}

Primary feasibility outcomes were determined by percent of recruitment, retention and adherence goals met. Study staff blinded to group assignment collected preliminary efficacy data on blood pressure, weight, and waist circumference. Blood pressure was measured as the mean of two measures taken five minutes apart using a mercury sphygmomanometer. Measurement of height (performed only at baseline) used a standard stadiometer and weight was measured and duplicated on a calibrated scale. Waist circumference was measured at the point of minimal circumference at the level of the uppermost lateral border of the right iliac crest at minimal respiration. In addition to the FBG and OGTT measurements, blood was also drawn for a fasting lipid panel and insulin. LDL was calculated directly. Fasting insulin was assessed using a Roche Automated Chemiluminescence analyzer Cobas e411 and Roche Diagnostic's insulin kits with an intra assay coefficient of variation $(\mathrm{CV})$ of $5.4 \%$ and inter assay $\mathrm{CV}$ of $6 \%$. We used the calculator available through the Diabetes Trials Unit to apply version 2 of the homeostasis model assessment (HOMA) algorithm to calculate insulin resistance [32].

Secondary outcomes related to psychological wellbeing included changes in depression, anxiety, positive affect, negative affect, and perceived stress. Depression and anxiety were measured using the Hospital Anxiety and Depression Scale (HADS), a 14-item questionnaire that avoids measuring symptoms such as fatigue that may be influenced by diabetes risk factors [33]. Positive and negative affect were measured using the Positive and Negative Affect Schedule (PANAS), a 20-item questionnaire designed to assess high activation positive affect (e.g. interested, excited, enthusiastic) and high activation negative affect (e.g. upset, irritable, ashamed) [34]. Stress was measured using the Perceived Stress Scale (PSS), a 10-item instrument asking participants how often they had positive and negative experiences related to stress in the prior month [35]. 


\section{Data analysis}

Within group changes and between group treatment effects associated with participation in the yoga intervention were evaluated using chi-square tests for categorical data and paired t-tests, independent sample t-tests, and Cohen's d effect sizes (standardized mean difference) for continuous data. Change scores were calculated as postintervention minus baseline measurements. Given the pilot nature of this study and the relatively small sample size, we did not have adequate power to detect statistically significant differences in the case of small or medium effect sizes. To provide a measure of the observed effect sizes, we report Cohen's d for primary and secondary outcome measures.

Three participants dropped out for personal reasons unrelated to the study. Based on this and the randomization, we assumed unobserved outcome data was missing at random (MAR) and therefore conducted two primary analyses. First, we excluded dropouts entirely in a complete-case analysis. With outcomes at one follow-up time point, complete case is a valid analysis assuming missing outcomes are MAR [36]. Second, we included dropouts with their last observation carried forward (LOCF) to include all randomized participants for ITT. In this case, the LOCF was the baseline value for the participant who dropped out. Because participants who dropped out did not complete the study, we also assumed that there was no change in their unobserved outcomes. Based on these two assumptions, the LOCF analysis provides a more conservative estimate of the within and between group effects [36].

We replicated the complete case, between group comparison in a per-protocol analysis, excluding the six participants who did not meet the FBG $\geq 5.6 \mathrm{mmol} / \mathrm{L}$ inclusion criteria. We calculated Pearson's correlation coefficients to further explore relationships between T2DM risk factors and psychological measures. In addition, based on the wide range of ages among enrolled participants (30-65 years) and the concern that a number of T2DM risk factors would vary based on age we conducted a sensitivity analysis to specifically examine whether baseline or change scores differed by age group. We considered $\mathrm{p}<0.05$ to be statistically significant and all analyses were conducted using Stata Statistical Software: Release 12. College Station, TX: StataCorp LP.

\section{Results}

Recruitment, retention and adherence goals to determine feasibility were all met in this pilot study. We retained $94 \%$ of participants, exceeding our goal for feasibility of $\geq 75 \%$ retention. On average, attendance each week for participants in the yoga group did not differ from attendance in the control group $(4 \pm 0.5$ yoga classes per week vs. $3.8 \pm$ 0.5 monitored walks per week, $\mathrm{p}=0.13$ ); however, participants in the yoga group had higher overall attendance compared to control $(\mathrm{p}=0.02)$. Participants in the yoga group attended $33.6 \pm 4.3$ classes over the eight weeks of the study, which is equivalent to $70 \%$ of the maximum offered (six classes per week) and 140\% of the minimum required (three classes per week). This is compared to attendance in the control group of $30.2 \pm 4.3$ walking sessions, which is equivalent to $63 \%$ of maximum walks offered and $126 \%$ of minimum walks required.

The two groups were similar at baseline. In the yoga group, the mean age was $47.0 \pm 9.7$ and $43 \%$ of participants were male compared to the control group where the mean age was $47.2 \pm 9.1$ and $35 \%$ of participants were male (Table 2). In between group comparisons, yoga participants had significantly greater reductions in weight, waist circumference and BMI versus the walking control participants (weight $-0.8 \pm 2.1$ vs. $1.4 \pm 3.6, \mathrm{p}=0.02$; waist circumference $-4.2 \pm 4.8$ vs. $0.7 \pm 4.2, \mathrm{p}>0.01$; BMI $-0.2 \pm 0.8$ vs. $0.6 \pm 1.6, \mathrm{p}=0.05$ ) (Table 3 ). There were no significant between group differences in FBG, PPBG, insulin, insulin resistance, blood pressure, cholesterol, or psychological measures of well-being.

There were, however, significant reductions in blood pressure and total cholesterol within both groups over the

Table 2 Baseline for Yoga and control groups as mean \pm SD unless indicated

\begin{tabular}{lcc}
\hline & $\begin{array}{c}\text { Yoga } \\
(\mathbf{n = 2 1 )}\end{array}$ & $\begin{array}{c}\text { Control } \\
(\mathbf{n}=\mathbf{2 0})\end{array}$ \\
\hline Age (years) & $47.0 \pm 9.7$ & $47.2 \pm 9.1$ \\
Gender (male), $n(\%)$ & $9(43)$ & $7(35)$
\end{tabular}

Diabetes risk factors

$\begin{array}{lcc}\text { Fasting BG (mmol/L) } & 6.4 \pm 0.9 & 6.7 \pm 1.9 \\ \text { Postprandial BG (mmol/L) } & 8.3 \pm 2.6 & 6.8 \pm 1.2 \\ \text { BMI (kg/m2) } & 28.4 \pm 5.3 & 26.9 \pm 3.0 \\ \text { Weight (kg) } & 69.2 \pm 10.8 & 65.0 \pm 7.6 \\ \text { Waist (cm) } & 92.5 \pm 7.3 & 89.8 \pm 7.2 \\ \text { Insulin (pmol/L) } & 72.3 \pm 30.5 & 57.7 \pm 30.1 \\ \text { Insulin resistance } & 1.4 \pm 0.6 & 1.1 \pm 0.6 \\ \text { Systolic (mmHg) } & 122.9 \pm 8.3 & 127.6 \pm 11.4 \\ \text { Diastolic (mmHg) } & 82.2 \pm 9.1 & 82.3 \pm 6.3 \\ \text { LDL (mmol/L) } & 2.2 \pm 0.5 & 2.2 \pm 0.8 \\ \text { Total cholesterol (mmol/L) } & 5.2 \pm 1.2 & 5.3 \pm 0.8 \\ \text { Triglycerides (mmol/L) } & 1.9 \pm 0.8 & 2.2 \pm 1.2 \\ \text { Psychological factors } & & \\ \text { Anxiety } & 7.8 \pm 5.3 & 8.3 \pm 5.2 \\ \text { Depression } & 6.0 \pm 4.0 & 6.8 \pm 3.8 \\ \text { Positive affect } & 24.9 \pm 7.7 & 22.4 \pm 11.2 \\ \text { Negative affect } & 15.4 \pm 12.7 & 17.3 \pm 11.6 \\ \text { Perceived stress } & 18.2 \pm 5.4 & 18.0 \pm 5.6\end{array}$

BG fasting blood glucose; BMI body mass index; LDL low-density lipoproteins. 
Table 3 Change scores within yoga and control, and difference between groups with $95 \% \mathrm{Cl}$ and Cohen's d effect size

\begin{tabular}{|c|c|c|c|c|c|}
\hline & $\begin{array}{c}\text { Yoga } \\
(n=20)\end{array}$ & $\begin{array}{l}\text { Control } \\
(n=18)\end{array}$ & $\begin{array}{l}\text { Diff. (Yoga-Ctrl) } \\
{[95 \% \mathrm{Cl}]}\end{array}$ & $\begin{array}{c}P \\
\text { diff. }\end{array}$ & $\begin{array}{c}\text { Cohen's } \\
\text { d }\end{array}$ \\
\hline \multicolumn{6}{|l|}{ Diabetes risk factors } \\
\hline Fasting BG (mmol/L) & 0 & -0.3 & $0.3[-0.5$ to 1.1$]$ & 0.43 & 0.27 \\
\hline Postprandial BG $(\mathrm{mmol} / \mathrm{L})^{a}$ & -0.6 & 0.1 & $-0.7[-2.4$ to 0.9$]$ & 0.38 & 0.37 \\
\hline BMI (kg/m2) & -0.2 & 0.6 & $-0.8[-1.6$ to -0.0$]$ & 0.05 & 0.68 \\
\hline Weight (kg) & -0.8 & 1.4 & $-2.3[-4.1$ to -0.4$]$ & 0.02 & 0.80 \\
\hline Waist (cm) & $-4.2^{*}$ & 0.7 & $-4.9[-7.9$ to -1.9$]$ & $<0.01$ & 1.11 \\
\hline Systolic (mmHg) & $-5.3^{*}$ & $-5.4^{*}$ & $0.1[-4.8$ to 5.1$]$ & 0.95 & 0.02 \\
\hline Diastolic (mmHg) & $-5.3^{*}$ & $-3.6^{*}$ & $-1.7[-7.6$ to 4.1$]$ & 0.55 & 0.20 \\
\hline Insulin (pmol/L) & -16.7 & 4.2 & $-20.9[-52.2$ to 10.4$]$ & 0.19 & 0.46 \\
\hline Insulin resistance & -0.3 & 0.1 & $-0.4[-1.0$ to 0.2$]$ & 0.17 & 0.48 \\
\hline LDL (mmol/L) & -0.1 & 0.0 & $-0.2[-0.6$ to 0.3$]$ & 0.47 & 0.24 \\
\hline Total cholesterol (mmol/L) & $-0.9^{*}$ & $-0.7^{*}$ & $-0.2[-0.9$ to 0.6$]$ & 0.67 & 0.15 \\
\hline Triglycerides (mmol/L) & -0.1 & -0.1 & $0[-0.6$ to 0.6$]$ & 0.95 & 0.02 \\
\hline \multicolumn{6}{|l|}{ Psychological factors $^{b}$} \\
\hline Anxiety & $-2.1^{*}$ & $-3.4^{*}$ & $1.3[-0.8$ to 3.4$]$ & 0.22 & 0.42 \\
\hline Depression & $-2.5^{*}$ & $-3.2^{*}$ & $0.7[-1.7$ to 3.2$]$ & 0.55 & 0.21 \\
\hline Positive affect & $4.8^{*}$ & 4.6 & $0.2[-4.9$ to 5.2$]$ & 0.95 & 0.02 \\
\hline Negative affect & $-5.0^{*}$ & $-8.4^{*}$ & $3.4[-2.3$ to 9.0$]$ & 0.24 & 0.41 \\
\hline Perceived stress & $-5.7^{*}$ & $-7.9^{*}$ & $2.2[-1.3$ to 5.8$]$ & 0.21 & 0.43 \\
\hline
\end{tabular}

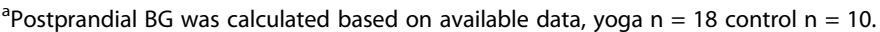

${ }^{\mathrm{b}}$ Three participants were missing data for psychological factors in the control group.

${ }^{*} p<0.05$.

BG blood glucose; BMI body mass index; LDL low density lipoproteins.

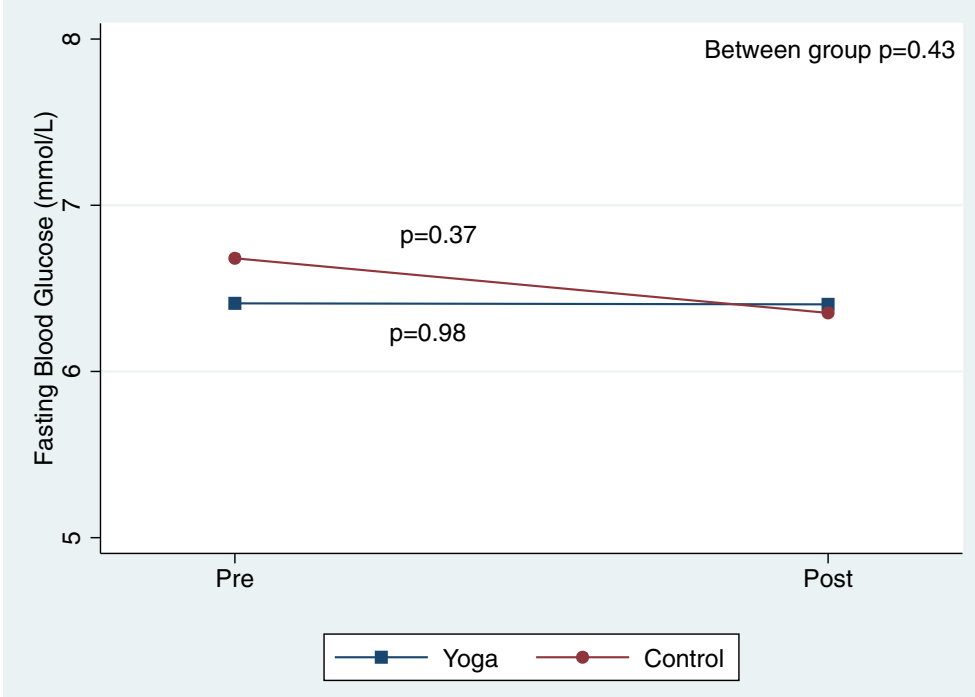

Figure 2 Pre/post changes in fasting blood glucose. 


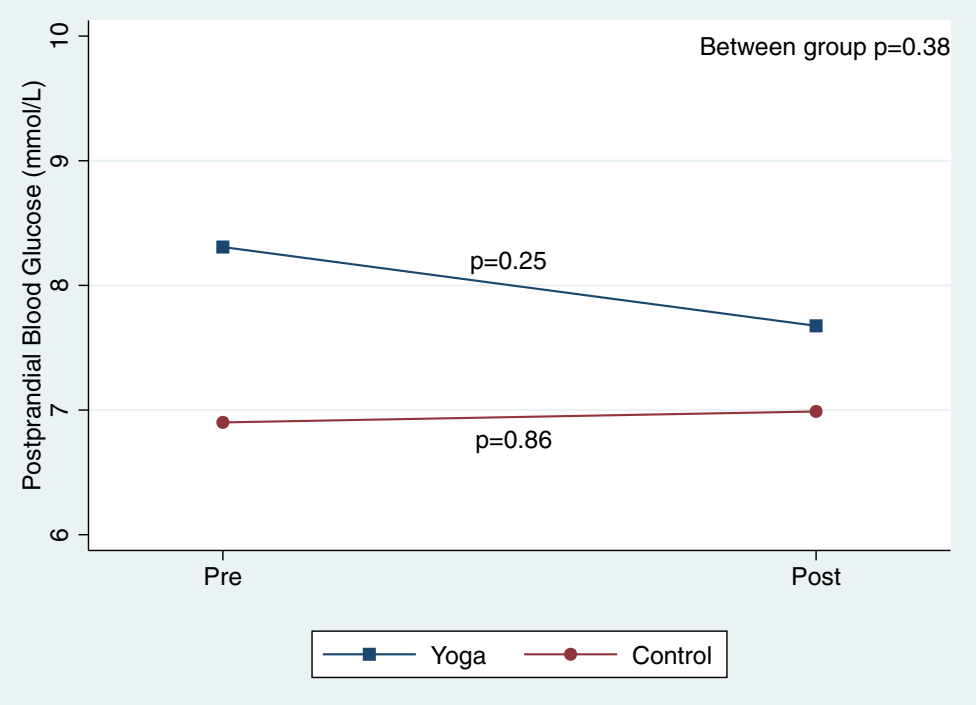

Figure 3 Pre/post changes in postprandial blood glucose.

course of the 8-week study (Table 3). Figures 2, 3, 4, 5, 6, $7,8,9,10,11,12$ and 13 illustrate within group changes in T2DM risk factors over time. There were also significant within group improvements in anxiety, depression, negative affect and perceived stress in both groups and a significant within group improvement in positive affect in the yoga group (Table 3). For LOCF analysis, the between group effects were not statistically or substantively different from those reported for the complete case analysis.

In the complete case per-protocol analysis, participants with screening FBG $<5.6 \mathrm{mmol} / \mathrm{L}$ were excluded (yoga $\mathrm{n}=$ 3 , control $n=3$ ). The between group reductions in weight, waist circumference and BMI continued to be significant and continued to favor the yoga intervention (Table 4). There were significant within group changes in systolic blood pressure in both groups, diastolic blood pressure in the control group and total cholesterol in the yoga intervention (Table 4). The within group changes in psychological factors in the per-protocol analysis were similar to those found in the main ITT analyses (Table 4).

Younger participants had significantly lower LDL and total cholesterol at baseline $(30-39$ years $1.6 \pm 0.5$; 40-49 years $2.2 \pm 0.6$; $50-59$ years $2.6 \pm 0.6, \geq 60$ years $2.39 \pm 0.4$, $\mathrm{p}=0.013$ ) but there were no differences in change scores

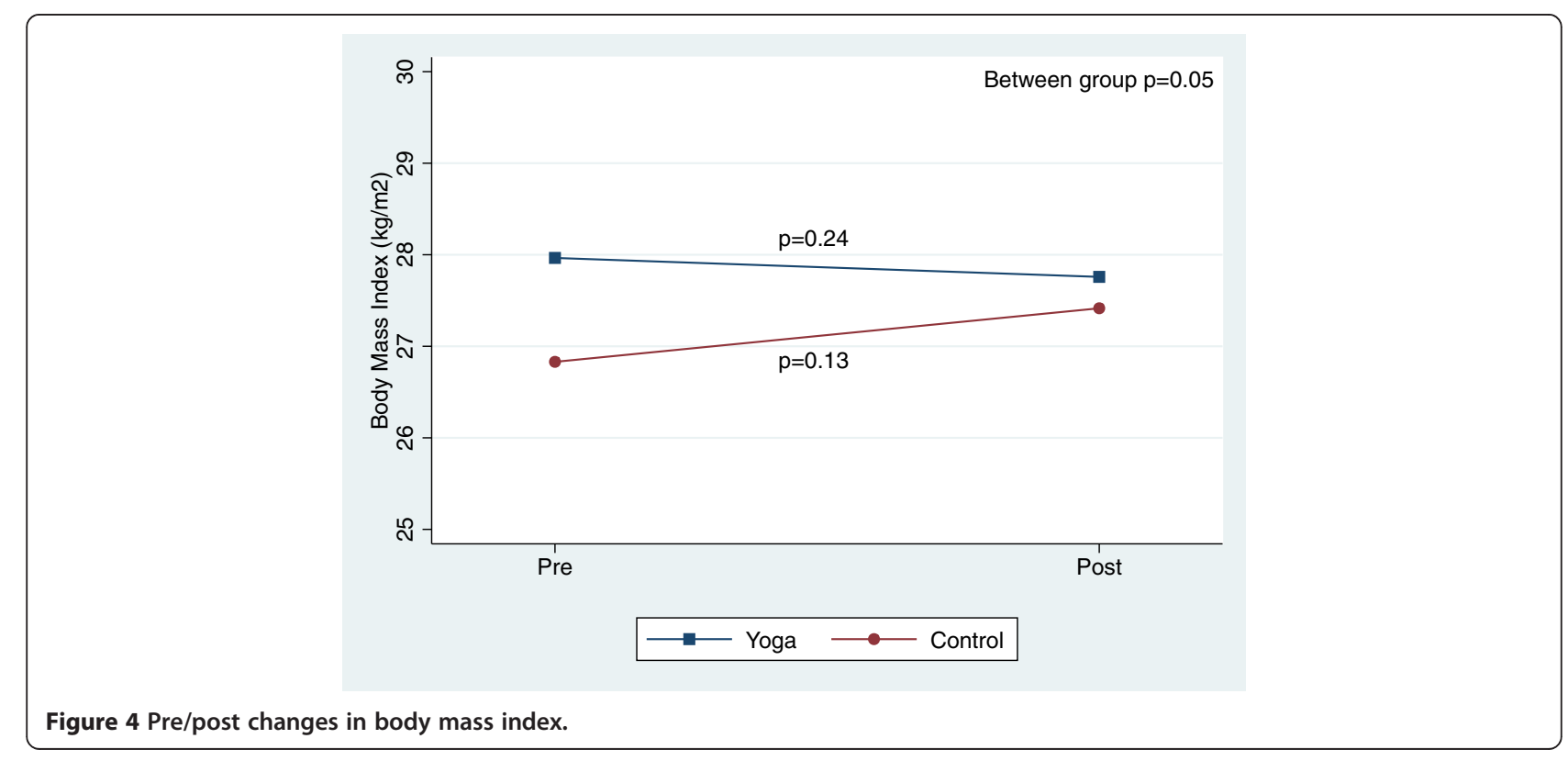




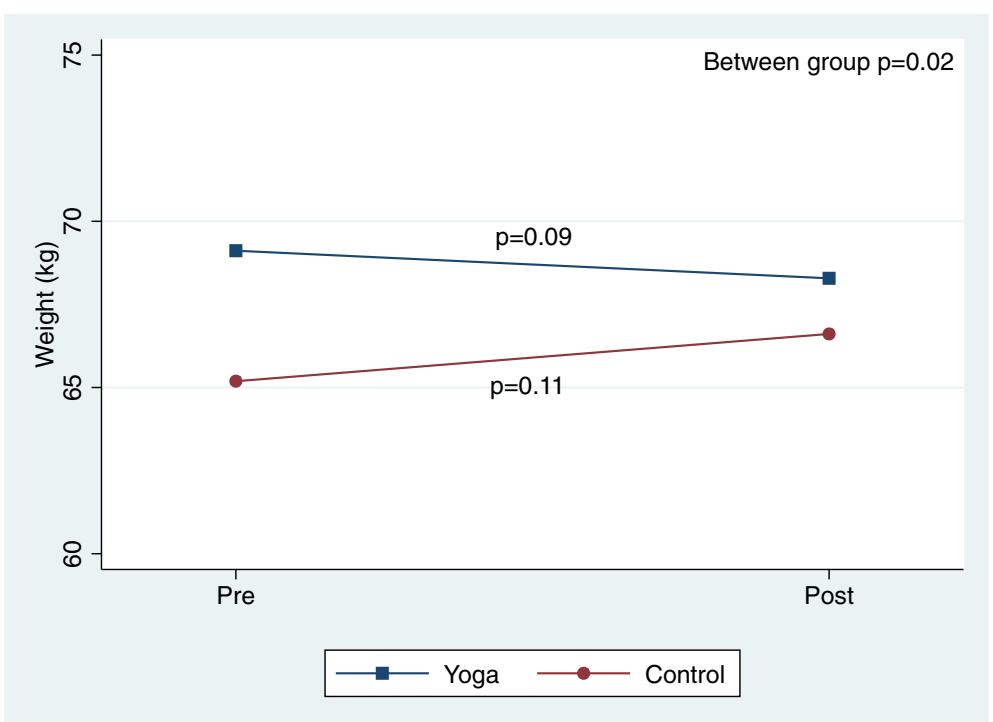

Figure 5 Pre/post changes in weight.

based on age. To explore potential relationships further, we examined correlations between changes in T2DM risk factors and psychological factors within the yoga group. We found significant correlations among changes in negative affect and PPBG $(\mathrm{r}=0.49, \mathrm{p}=0.03)$ and changes in perceived stress and total cholesterol $(\mathrm{r}=-0.47, \mathrm{p}=0.04)$. We also saw trends for correlations between changes in depression and PPBG $(\mathrm{r}=0.46, \mathrm{p}=0.06)$; positive affect and insulin $(\mathrm{r}=0.40, \mathrm{p}=0.08)$; perceived stress and BMI $(\mathrm{r}=0.42, \mathrm{p}=0.06)$; perceived stress and weight $(\mathrm{r}=0.43$, $\mathrm{p}=0.06)$; and positive affect and insulin resistance $(\mathrm{r}=$ $0.42, \mathrm{p}=0.07)$.

\section{Discussion}

Both the yoga intervention and walking control groups in this study exceeded recruitment, retention and adherence goals, meeting feasibility criteria. We found a significant decrease in weight, BMI and waist circumference in the yoga group compared to the walking control, however we did not see a decrease in FBG, PPBG, insulin, insulin resistance, blood pressure, or cholesterol. We did find significant within group decreases in blood pressure and total cholesterol in both yoga and walking control groups as well as within group improvements in measures of psychological well-being.

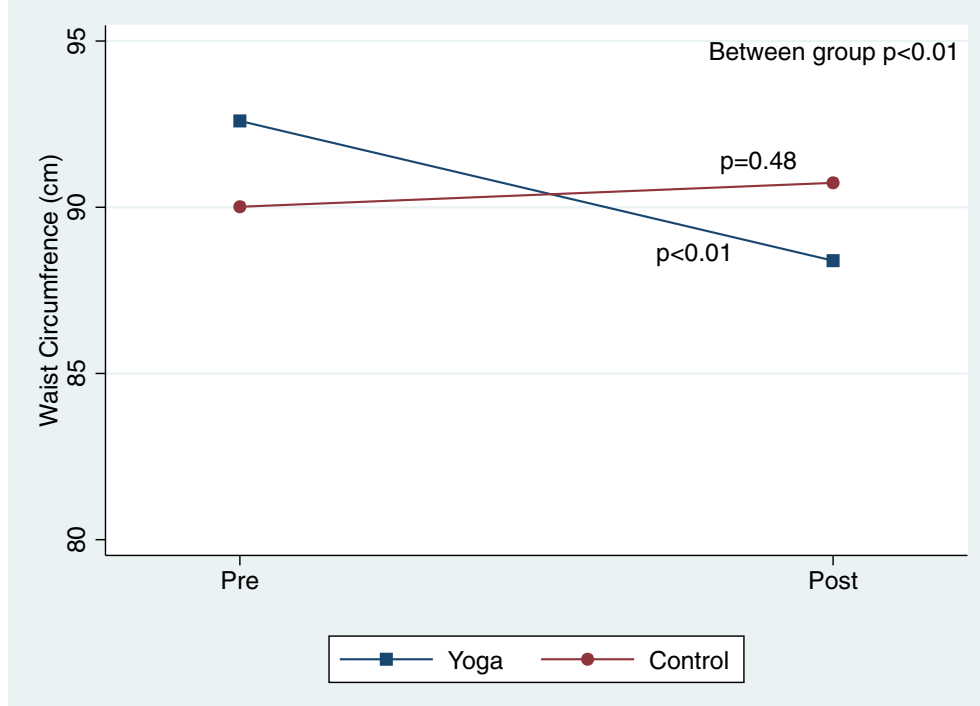

Figure 6 Pre/post changes in waist circumference. 


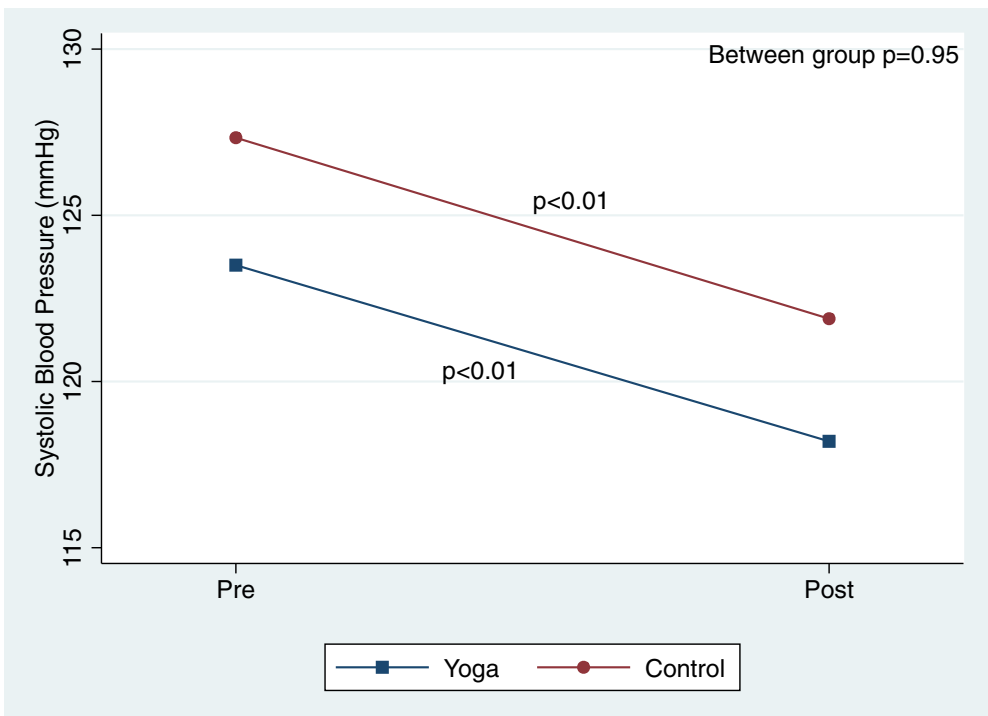

Figure 7 Pre/post changes in serum insulin.

In the yoga group, positive changes in weight, BMI and waist circumference are similar to other reports in the literature $[23,25,37]$, while the lack of within group change in FBG is contrary to other reports in similar high risk populations $[23,24]$. In the per-protocol analysis we excluded participants with very low baseline FBG (those in the normal range) and still saw no change in FBG. Our null results were similar to another small study of yoga for high risk individuals (those with a first degree relative with T2DM plus one of the following: impaired FBG, prehypertension, obesity, or abnormal cholesterol) [25] supporting the notion that we may have lacked power to detect small to medium FBG changes. Over time, improvements in weight and waist circumference could yield additional improvements in FBG and related measures.

While both groups had statistically significant within group improvements in measures of psychological wellbeing, there were no significant differences between groups. The control group likely experienced significant improvement in psychological well-being based on the walking that they were asked to participate in three to six days a week. While we had initially hypothesized that the yoga would improve mood based on a number of factors,

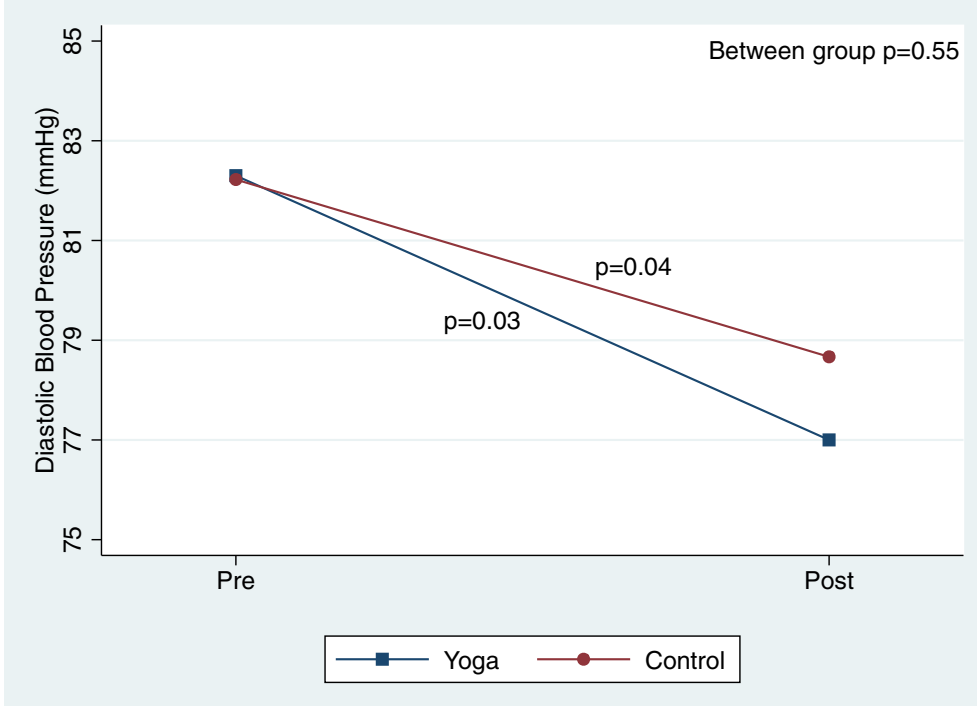

Figure 8 Pre/post changes in insulin resistance. 


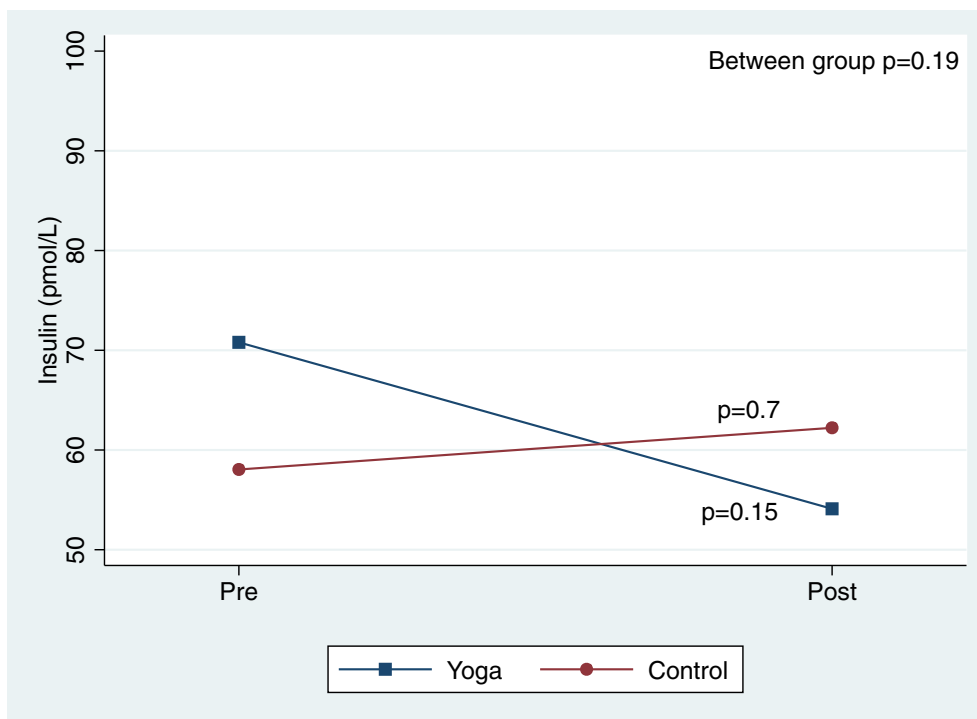

Figure 9 Pre/post changes in systolic blood pressure.

the effect of increased walking in the control group appears to have had an equally powerful effect. There was a significant within group increase in positive affect in the yoga group and not in the walking control, however, the between group difference was not significant. In a recent large randomized controlled trial, investigators found that increasing positive affect significantly improved physical activity maintenance at 12 months suggesting a potential behavior change advantage for the yoga group [38].
There are several important limitations in our study. First, several participants did not complete the OGTT, resulting in missing data and limiting our use of OGTT to establish initial eligibility. Second, six participants with borderline FBG did not meet the inclusion criteria of FBG $\geq 5.6 \mathrm{mmol} / \mathrm{L}$ but were mistakenly enrolled and randomized in this study. By including these participants, we are not addressing our original research question whether yoga reduces T2DM risk among participants with

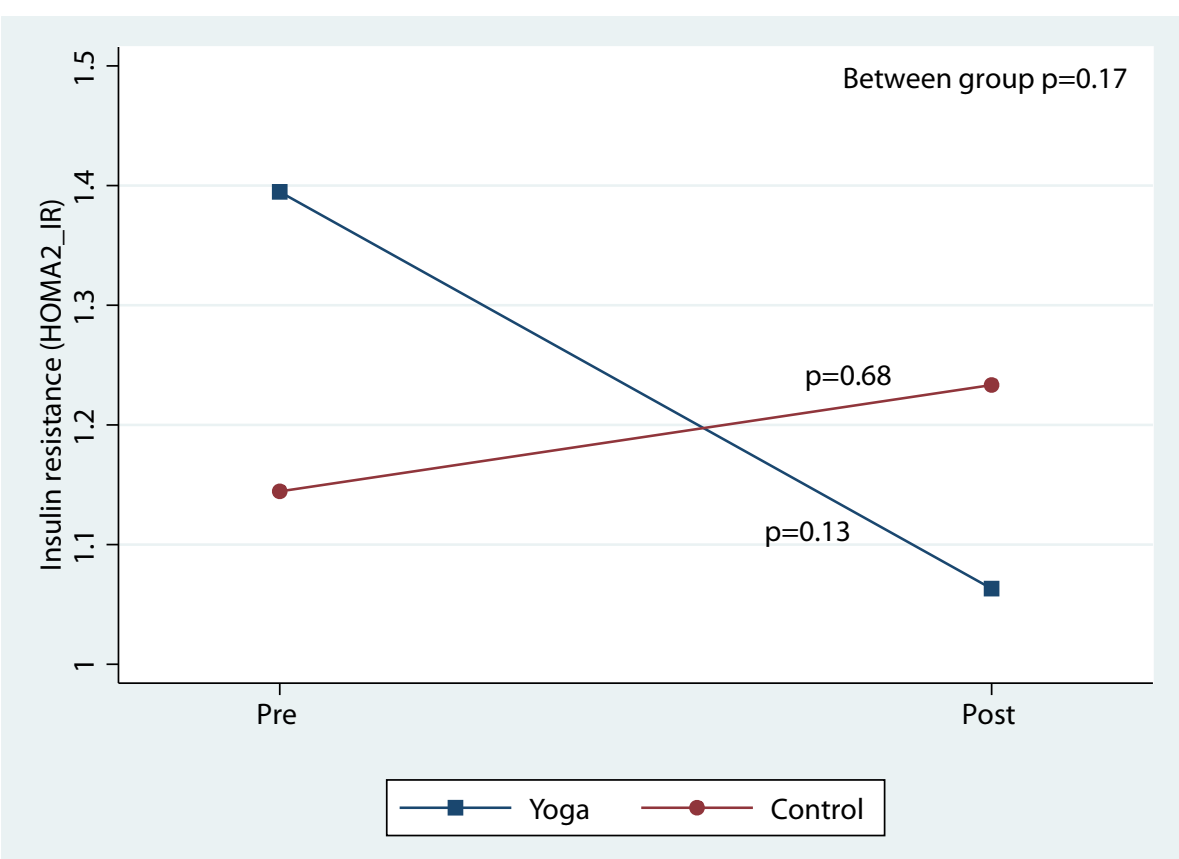

Figure 10 Pre/post changes in diastolic blood pressure. 


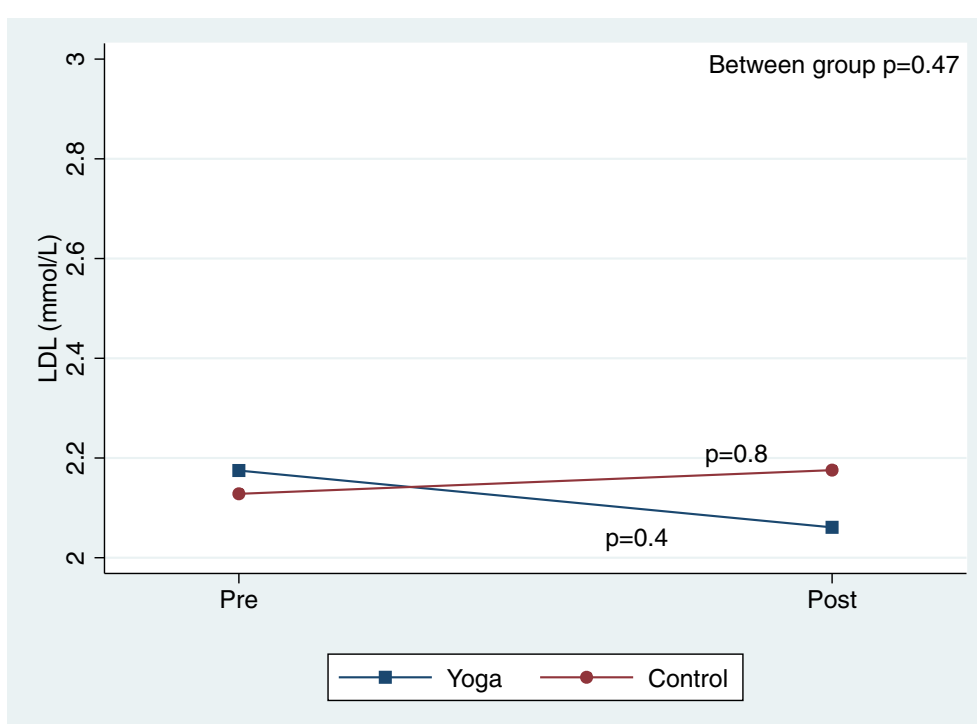

Figure 11 Pre/post changes in low-density lipoproteins.

FBG $\geq 5.6 \mathrm{mmol} / \mathrm{L}$. To address this, we also included a per-protocol analysis excluding the six participants with FBG $<5.6 \mathrm{mmol} / \mathrm{L}$. Third, this study was conducted in India where yoga originated and continues to maintain significant cultural influence. This potentially limits the generalizability of feasibility and adherence findings outside of India. Finally, given the modest sample size in this pilot study, we were not able to detect differences other than large effects.

\section{Conclusions}

In summary, our study has several implications for future investigations of yoga-based interventions for individuals at high risk of T2DM. Our results indicate that yoga is a feasible intervention strategy and may help reduce weight, BMI and waist circumference, three important factors in T2DM risk. We did not find clear evidence of benefit in FBG, PPBG, or insulin resistance however, over time, improvements in weight and waist circumference could yield

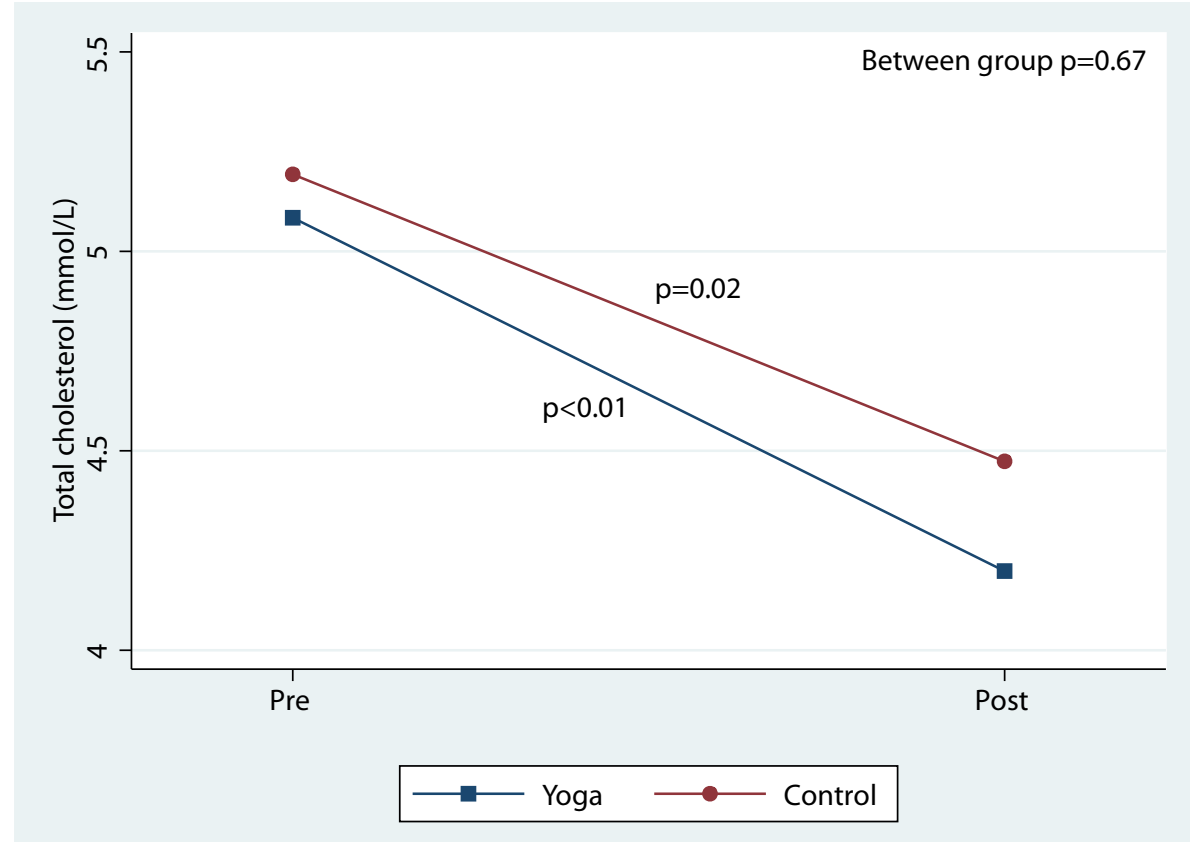

Figure 12 Pre/post changes in total cholesterol. 


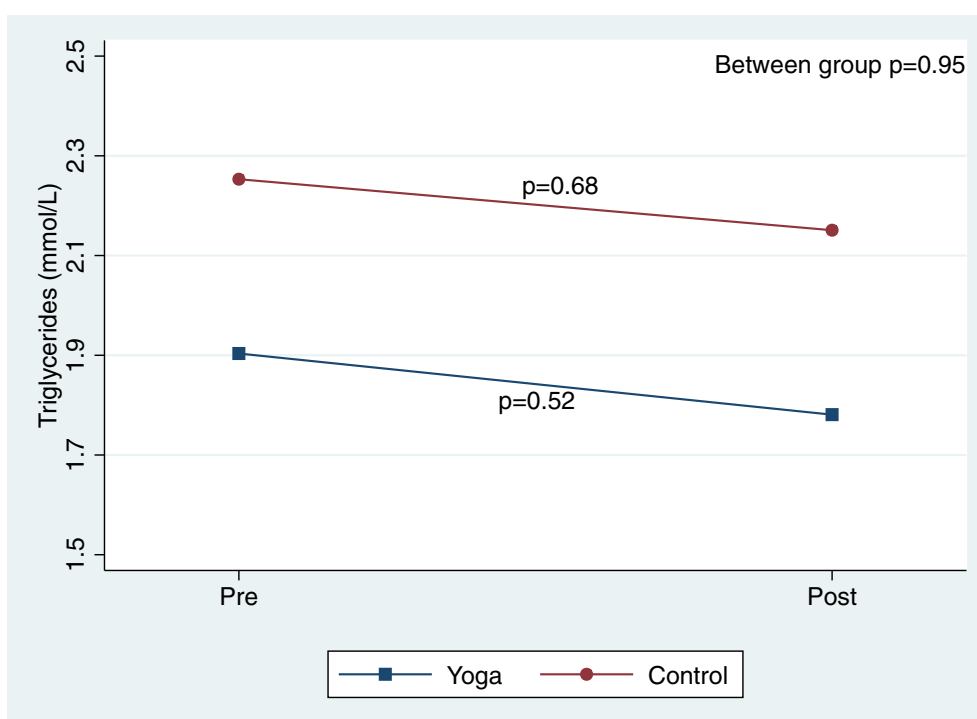

Figure 13 Pre/post changes in triglycerides.

Table 4 Per-protocol ${ }^{\mathrm{a}}$ change scores within yoga and control, and difference between groups with $95 \% \mathrm{Cl}$ and $\mathrm{Cohen}$ 's d effect size

\begin{tabular}{|c|c|c|c|c|c|}
\hline & $\begin{array}{c}\text { Yoga } \\
n=17\end{array}$ & $\begin{array}{l}\text { Control } \\
n=15\end{array}$ & $\begin{array}{c}\text { Diff. (Yoga- Ctrl) } \\
{[95 \% \mathrm{Cl}]}\end{array}$ & $P$ diff. & Cohen's D \\
\hline \multicolumn{6}{|l|}{ Diabetes risk factors } \\
\hline Fasting BG (mmol/L) & 0 & -0.5 & $0.5[-0.5$ to 1.4$]$ & 0.32 & 0.37 \\
\hline Postprandial BG $(\mathrm{mmol} / \mathrm{L})^{\mathrm{b}}$ & -0.4 & 0.3 & $-0.7[-2.8$ to 1.4$]$ & 0.52 & 0.32 \\
\hline BMI (kg/m2) & -0.2 & 0.6 & $-0.9[-1.8$ to 0.1$]$ & 0.07 & 0.69 \\
\hline Weight (kg) & -1.0 & 1.5 & $-2.5[-4.8$ to -0.3$]$ & 0.03 & 0.83 \\
\hline Waist (cm) & $-4.9^{*}$ & 0.7 & $-5.6[-8.9$ to -2.2$]$ & $<0.01$ & 1.24 \\
\hline Systolic (mmHg) & $-4.4^{*}$ & $-6.5^{*}$ & $2.2[-3.0$ to 7.4$]$ & 0.40 & 0.31 \\
\hline Diastolic (mmHg) & -4.6 & $-3.9^{*}$ & $-0.7[-7.1$ to 5.6$]$ & 0.82 & 0.08 \\
\hline Insulin (pmol/L) & -14.6 & 4.1 & $-18.7[-54.6$ to 17.3$]$ & 0.3 & 0.39 \\
\hline Insulin resistance & -0.3 & 0.1 & $-0.4[-1.1$ to 0.3$]$ & 0.28 & 0.40 \\
\hline $\mathrm{LDL}(\mathrm{mmol} / \mathrm{L})$ & -0.1 & 0.2 & $-0.3[-0.8$ to 0.1$]$ & 0.17 & 0.52 \\
\hline Total cholesterol (mmol/L) & $-0.9^{*}$ & -0.7 & $-0.2[-1.1$ to 0.6$]$ & 0.58 & 0.21 \\
\hline Triglycerides (mmol/L) & -0.2 & -0.3 & $0.1[-0.6$ to 0.7$]$ & 0.83 & 0.08 \\
\hline \multicolumn{6}{|l|}{ Psychological factors ${ }^{c}$} \\
\hline Anxiety & $-1.7^{*}$ & $-3.1^{*}$ & $1.4[-0.7$ to 3.6$]$ & 0.19 & 0.50 \\
\hline Depression & $-2.3^{*}$ & $-3.1^{*}$ & $0.8[-1.9$ to 3.4$]$ & 0.56 & 0.22 \\
\hline Positive affect & $4.8^{*}$ & 4.7 & $0.1[-5.8$ to 5.9$]$ & 0.99 & 0.01 \\
\hline Negative affect & $-4.5^{*}$ & $-7.6^{*}$ & $3.0[-2.4$ to 8.5$]$ & 0.27 & 0.42 \\
\hline Perceived stress & $-6.3^{*}$ & $-8.1^{*}$ & $1.8[-1.7$ to 5.3$]$ & 0.31 & 0.38 \\
\hline
\end{tabular}

${ }^{a}$ Six participants were excluded (yoga $n=3$; control $n=3$ ) based on screening FBG $<5.6$.

${ }^{\mathrm{b}}$ Postprandial BG was calculated based on available data, yoga $n=17$ control $n=7$.

'One participant was missing data for psychological factors in the control group.

${ }^{*} \mathrm{p}<0.05$.

BG blood glucose; BMI body mass index; LDL low density lipoproteins. 
additional improvements in these parameters. To further test the potential benefits of yoga for T2DM prevention, our results suggest that studies of longer duration and larger sample size are important.

\section{Abbreviations}

BMI: Body mass index; Cl: Confidence intervals; FBG: Fasting blood glucose; HADS: Hospital Anxiety and Depression Scale; HOMA: Homeostatic model assessment (version 2): METs: Metabolic equivalent of task: NCCAM: National Center for Complementary and Alternative Medicine; OCIM: Osher Center for Integrative Medicine; OGTT: Oral glucose tolerance test; PANAS: Positive and Negative Affect Schedule; PPBG: Postprandial blood glucose; PSS: Perceived Stress Scale; SVYASA: Swami Vivekananda Yoga Anusandhana Samsthana; T2DM: Type 2 diabetes mellitus; UCSF: University of California, San Francisco.

\section{Competing interests}

The authors declare that they have no competing interests.

\section{Authors' contributions}

$F M H, M R R, R N, E J M, A B$, and RHN conceived of the study and participated in design and coordination of preliminary work. MRR, RN and RHN conducted and oversaw staff functions in recruitment, screening and primary data collection in Bangalore, India. KAM, MRR, and FMH analyzed the data and drafted the manuscript. All authors read and approved the manuscript.

\section{Acknowledgements}

This study was supported with a grant from the US National Institutes of Health, NCCAM R21 AT01942. Dr. McDermott's time was supported by NCCAM T32 AT003997, and Dr. Hecht's time was supported by NCCAM K24 AT007827.

\section{Funding}

This study was supported with a grant from the US National Institutes of Health, NCCAM R21 AT01942.

\section{Author details}

'Osher Center for Integrative Medicine, University of California, 1545 Divisadero St., San Francisco, CA 94115, USA. ${ }^{2}$ Swami Vivekananda Yoga Anusandhana Samsthana, Bangalore, India. ' ${ }^{3}$ epartment of Medicine, Division of Endocrinology and Metabolism, University of California, San Francisco, CA, USA. ${ }^{4}$ Institute for Holistic Health Studies, San Francisco State University, San Francisco, CA, USA.

Received: 25 September 2013 Accepted: 23 June 2014 Published: 1 July 2014

\section{References}

1. Whiting DR, Guariguata L, Weil C, Shaw J: IDF Diabetes Atlas: Global estimates of the prevalence of diabetes for 2011 and 2030. Diabetes Res Clin Pract 2011, 94:311-321.

2. World Health Organization: International Diabetes Federation: Definition and diagnosis of diabetes mellitus and intermediate hyperglycemia: report of a WHO/IDF Consultation. Geneva: World Health Organization; 2006.

3. Desai $\mathrm{A}$, Tandon $\mathrm{N}$ : Challenges in prevention and management of diabetes mellitus and metabolic syndrome in India. Curr Sci 2009, 97:356-366.

4. Misra A, Vikram NK: Insulin resistance syndrome (metabolic syndrome) and obesity in Asian Indians: evidence and implications. Nutrition 2004, 20:482-491

5. Gupta A, Gupta R, Sarna M, Rastogi S, Gupta VP, Kothari K: Prevalence of diabetes, impaired fasting glucose and insulin resistance syndrome in an urban Indian population. Diabetes Res Clin Pract 2003, 61:69-76.

6. Nathan DM, Davidson MB, DeFronzo RA, Heine RJ, Henry RR, Pratley R, Zinman B, American Diabetes Association: Impaired fasting glucose and impaired glucose tolerance: implications for care. Diabetes Care 2007, 30:753-759.

7. Expert Panel on Detection, Evaluation, and Treatment of High Blood Cholesterol in Adults: Executive Summary of The Third Report of The National Cholesterol Education Program (NCEP) Expert Panel on Detection, Evaluation, And Treatment of High Blood Cholesterol In Adults (Adult Treatment Panel III). JAMA 2001, 285:2486-2497.
8. Misra A, Wasir JS, Pandey RM: An Evaluation of Candidate Definitions of the Metabolic Syndrome in Adult Asian Indians. Diabetes Care 2005, 28:398-403.

9. Tuomilehto J, Lindström J, Eriksson JG, Valle TT, Hämäläinen $H$, Ilanne-Parikka P, Keinänen-Kiukaanniemi S, Laakso M, Louheranta A, Rastas M: Prevention of type 2 diabetes mellitus by changes in lifestyle among subjects with impaired glucose tolerance. New Engl J Med 2001, 344:1343-1350.

10. Boulé NG, Haddad E, Kenny GP, Wells GA, Sigal RJ: Effects of exercise on glycemic control and body mass in type 2 diabetes mellitus: a metaanalysis of controlled clinical trials. JAMA 2001, 286:1218-1227.

11. Zanuso S, Jimenez A, Pugliese G, Corigliano G, Balducci S: Exercise for the management of type 2 diabetes: a review of the evidence. Acta Diabetol 2009, 47:15-22.

12. Richter EA, Derave W: Glucose, exercise and insulin: emerging concepts. J Physiol 2001, 535:313-322.

13. Blair SN, Horton E, Leon AS, Lee I-M, Drinkwater BL, Dishman RK, Mackey M, Kienholz ML: Physical activity, nutrition, and chronic disease. Med Sci Sports Exerc 1996, 28:335-349.

14. Katzmarzyk PT, Leon AS, Wilmore JH, Skinner JS: Targeting the Metabolic Syndrome with Exercise: Evidence From the HERITAGE Family Study. Med Sci 2003, 35:1703-1709.

15. Laaksonen DE, Lindström J, Lakka TA, Eriksson JG, Niskanen L, Wikström K, Aunola S, Keinänen-Kiukaanniemi S, Laakso M, Valle TT, Ilanne-Parikka P, Louheranta A, Hämäläinen H, Rastas M, Salminen V, Cepaitis Z, Hakumäki M, Kaikkonen H, Härkönen P, Sundvall J, Tuomilehto J, Uusitupa M, Finnish diabetes prevention study: Physical activity in the prevention of type 2 diabetes: the Finnish diabetes prevention study. Diabetes 2005, 54:158-165.

16. lyengar B: Light on Yoga: Yoga Dipika. New York: Schocken Books, Inc; 1995.

17. Svatmarama, Akers BD: The Hatha Yoga Pradipika. Woodstock: YogaVidya. com; 2002.

18. Danucalov MÁD, Simões RS, Kozasa EH, Leite JR: Cardiorespiratory and Metabolic Changes during Yoga Sessions: The Effects of Respiratory Exercises and Meditation Practices. Appl Psychophysiol Biofeedback 2008, 33:77-81.

19. Ray US, Pathak A, Tomer OS: Hatha Yoga Practices: Energy Expenditure. Respiratory Changes and Intensity of Exercise. Evidence-Based Complement Altern Med 2011, 2011:1-12.

20. Hagins $M$, Moore W, Rundle A: Does practicing hatha yoga satisfy recommendations for intensity of physical activity which improves and maintains health and cardiovascular fitness? BMC Complement Altern Med 2007, 7:40.

21. Ross $A$, Thomas $S$ : The health benefits of yoga and exercise: a review of comparison studies. J Altern Complement Med 2010, 16:3-12.

22. Nagarathna R, Usharani MR, Rao AR, Chaku R, Kulkarni R, Nagendra HR: Efficacy of yoga based life style modification program on medication score and lipid profile in type 2 diabetes-a randomized control study. Int J Diabetes Dev Ctries 2012, 32:122-130.

23. Innes K, Bourguignon C, Taylor A: Risk indices associated with the insulin resistance syndrome, cardiovascular disease, and possible protection with yoga: A systematic review. J Am Board Fam Pract 2005, 18:491-519.

24. Kanaya AM, Araneta MRG, Pawlowsky SB, Barrett-Connor E, Grady D, Vittinghoff E, Schembri M, Chang A, Lou Carrion-Petersen M, Coggins T, Tanori D, Armas JM, Cole RJ: Restorative yoga and metabolic risk factors: The Practicing Restorative Yoga vs. Stretching for the Metabolic Syndrome (PRYSMS) randomized trial. J Diabetes Complicat 2014, 28:406-412.

25. Yang K, Bernardo LM, Sereika SM, Conroy MB, Balk J, Burke LE: Utilization of 3-Month Yoga Program for Adults at High Risk for Type 2 Diabetes: A Pilot Study. Evidence-Based Complement Altern Med 2011, 2011:1-6.

26. Jyotsna V, Ambekar S, Dhawan A, Joshi A, Kumar N, Sreenivas V: Comprehensive yogic breathing program improves quality of life in patients with diabetes. Indian J Endocr Metab 2012, 16:423.

27. Alexander GK, Innes KE, Selfe TK, Brown CJ: "'More than I expected"': Perceived benefits of yoga practice among older adults at risk for cardiovascular disease. Complement Ther Med 2013, 21:14-28.

28. Kosuri M, Sridhar GR: Yoga Practice in Diabetes Improves Physical and Psychological Outcomes. Metab Syndr Relat Disord 2009, 7:515-518.

29. Sahay BK: Yoga and diabetes. J Assoc Physicians India 1986, 34:645-648.

30. Telles $S$, Nagarathna R, Nagendra HR: Breathing through a particular nostril can alter metabolism and autonomic activities. Indian J Physiol Pharmacol 1994, 38:133-137. 
31. Telles S, Nagarathna R, Nagendra HR: Physiological measures of right nostril breathing. J Altern Complement Med 1996, 2:479-484.

32. Wallace TM, Levy JC, Matthews DR: Use and abuse of HOMA modeling. Diabetes Care 2004, 27:1487-1495.

33. Zigmond AS, Snaith RP: The hospital anxiety and depression scale. Acta Psychiatr Scand 1983, 67:361-370.

34. Watson D, Clark LA, Tellegen A: Development and validation of brief measures of positive and negative affect: the PANAS scales. $J$ Pers Soc Psychol 1988, 54:1063-1070.

35. Cohen S: Perceived Stress in a Probability Sample of the United States. In The social psychology of health: Claremont Symposium on applied social psychology. Edited by Spacapan S, Oskamp S. Newbury Park, CA: Sage Publications; 1988:31-67.

36. White IR, Carpenter J, Horton NJ: Including all individuals is not enough: lessons for intention-to-treat analysis. Clin Trials 2012, 9:396-407.

37. Seo DY, Lee S, Figueroa A, Kim HK, Baek YH, Kwak YS, Kim N, Choi TH, Rhee BD, Ko KS, Park BJ, Park SY, Han J: Yoga Training Improves Metabolic Parameters in Obese Boys. Korean J Physiol Pharmacol 2012, 16:175.

38. Peterson JC, Charlson ME, Hoffman Z, Wells MT, Wong SC, Hollenberg JP, Jobe JB, Boschert KA, Isen AM, Allegrante JP: A Randomized Controlled Trial of Positive-Affect Induction to Promote Physical Activity After Percutaneous Coronary Intervention. Arch Intern Med 2012, 172:329-336.

doi:10.1186/1472-6882-14-212

Cite this article as: McDermott et al: A yoga intervention for type 2 diabetes risk reduction: a pilot randomized controlled trial. $B M C$

Complementary and Alternative Medicine 2014 14:212.

\section{Submit your next manuscript to BioMed Central and take full advantage of:}

- Convenient online submission

- Thorough peer review

- No space constraints or color figure charges

- Immediate publication on acceptance

- Inclusion in PubMed, CAS, Scopus and Google Scholar

- Research which is freely available for redistribution 